\title{
Amplify-forward and decode-forward cooperation relying on systematic Luby transform coded Hybrid Automatic-Repeat-reQuest
}

\author{
H.A. Ngo T.D. Nguyen L. Hanzo \\ School of Electronics \& Computer Science, University of Southampton, Southampton, UK \\ E-mail:Ih@ecs.soton.ac.uk
}

\begin{abstract}
Systematic Luby transform (SLT) codes constitute rateless codes, which are capable of adaptively adjusting their code rate depending on the channel quality without any explicit channel state information (CSI) at the transmitters. SLTs are also suitable for space time collaboration-aided relay networks. In this study, an iterative decoding-aided SLT scheme is combined with 16 quadrature amplitude modulation (QAM) transmission in a wireless relay-aided network. The bit error ratio (BER) results and extrinsic information transfer (EXIT) charts are provided to evaluate the performance of the proposed scheme. The simulation results show that the proposed scheme using amplify-and-forward (AF) relaying achieves a $2.5 \mathrm{~dB}$ gain at a BER of $10^{-5}$, while the attainable improvement is nearly $6 \mathrm{~dB}$ for decode-and-forward (DF) relaying, compared to the noniterative detection scheme operating without the relay's assistance. Moreover, the AF relaying-aided SLT coded 16-QAM scheme is more beneficial, when the relay station is close to the source. By contrast, the DF relaying performs best near the mid-point between the source and the destination. Additionally, a modified Hybrid Automatic-Repeat-reQuest (HARQ) protocol using incremental redundancy is applied along with the SLT-coded 16-QAM scheme in order to enhance the achievable throughput and energy efficiency of cooperative networks. This arrangement reduces the total transmit power by about $5 \%$, compared to the classic HARQ scheme.
\end{abstract}

\section{Introduction}

Hybrid Automatic Repeat reQuest (HARQ) techniques are capable of supporting reliable data transmission over the wireless channels. This technique was introduced in the 1960s by Wozencraft and Horstein [1, 2], both for error detection and error correction combined with retransmission requests. Their system is now known as the Type-I HARQ. Naturally, the combination of forward-error-correction (FEC) codes and the classic ARQ protocol is capable of improving the achievable throughput and of reducing the number of retransmissions, hence the delay. An improved version of this system, known as the Type-II HARQ, was invented by $\mathrm{Lin}$ and $\mathrm{Yu}$ [3]. The HARQ is widely employed in contemporary communication systems, such as the Universal Mobile Telecommunications System (UMTS) and the 3GPP long-term evolution (LTE) standards [4] or in the IEEE 802.16 mobile WiMAX [5].

Diverse FEC schemes may be employed in HARQ systems. For example, systematic Luby transform (SLT) codes [6] are powerful erasure-filling codes and hence they substantially reduce the number of HARQ retransmissions. However, the coding scheme has not been thoroughly investigated, especially when collaborating with other system's component, such as the demodulator, using softinformation exchanging. Hence, the SLT using iterative detection (TD) will be studied along with the ARQ protocol. The proposed configuration will be referred to as the HARQ SLT scheme.

Multiple-input-multiple-output (MIMO) techniques [7] have been adopted for several wireless standard, such as the WiFi 802.11n and WiMax 802.16e schemes [5]. In principle, MIMO systems [7] use multiple antennas in different configurations combined with advanced coding schemes for improving the achievable performance, including the throughput and cellular coverage area. However, mobile handsets are constrained both in term of their size and battery power. Hence, their ability to accommodate MIMO elements is limited. This problem may be circumvented by the sophisticated concerted activation of the single antennas of multiple mobile stations as in amplify-and-forward (AF), decode-and-forward (DF) as well as compress-and-forward (CF) cooperation. More specifically, Sendonaris et al. [8, 9] demonstrated that both the throughput and coverage area may be simultaneously increased by employing a simple AF cooperation scheme. The DF and CF cooperative schemes were studied in $[10,11]$, demonstrating that the DF strategy is capable of achieving rates approaching the ergodic capacity of the channel.

The above-mentioned virtual MIMO concept was introduced by Dohler et al. [12]. Other MIMO techniques, such as space-time-coded cooperation (STCC) [13] and double space-time-coded cooperation [14] are also capable of providing substantial system performance benefits. 
Zhang et al. [15] proposed using the classic turbo code concept for cooperative communications, while $\mathrm{Ng}$ et al. [16] designed distributed turbo trellis-coded modulation using iterative soft-decoding. The performance of rateless codes, such as LT and Raptor codes, were compared with punctured codes in [17] and were further investigated in a wireless relay-aided network in [18]. Another coding scheme designed for cooperative communication is constituted by the HARQ scheme of $[19,20]$.

The rateless nature of SLT codes is attractive in hybridARQ systems [3], because they can deliver exactly the required amount of redundancy for ensuring error-free decoding. Naturally, this statement is only valid, if the transmission-regime used has a 'perfect granularity', that is, allows the transmission of a single extra symbol, when needed for successful decoding. This scenario was considered, for example, in [21], where near-capacity operation was achieved across a wide range of signal-tonoise ratio (SNRs). These benefits may also be directly exploited in cooperative systems. In this paper, we adopted a more practically motivated approach, where a more 'coarse retransmission granularity' is considered, since in realistic wireless systems it is infeasible to transmit a single symbol. This is because using transmission bursts of a few hundred symbols facilitate convenient channel estimation and synchronisation with the aid of a reasonable pilotoverhead, hence justifying the employment of the limited number of code rates ranging from $1 / 3$ to $1 / 2$.

All these above-mentioned techniques play an important role in the overall system designs, but they have typically been investigated independently. Therefore in this paper we proposed an intrinsically amalgamated HARQ-aided SLTcoded 16-QAM scheme for employment in relay-aided networks to investigate the interplay of these techniques on the performance of the entire system. The novelty and rationale of our proposed scheme is summarised as follows:

1. First, we contrived a novel system based on the concatenation of SLT codes and 16-QAM, where the decoder and the demodulator iteratively exchange extrinsic information. We will demonstrate that the arrangement is capable of providing a significant coding gain for transmission over wireless Rayleigh fading channels, compared to the system where the SLT coding scheme and the demodulator operate independently.

2. Furthermore, a sophisticated HARQ-aided SLT-coded 16QAM scheme using ID was introduced in a relay-aided wireless network. The proposed scheme provides an increased spatial diversity for the signals received at the destination. As a result, the achievable bit error ratio (BER) performance is enhanced. Moreover, we investigate the best transmit power sharing between the source and the relay stations (RS) and determine the best location both for AF and DF relaying schemes.

3. Finally, we improved the HARQ-aided SLT-coded 16QAM scheme's performance, despite reducing the number of incremental redundancy transmissions required. Explicitly, we reduced the system's total transmit power and increased the attainable throughput. Where possible, analytical expressions are provided for characterising the system.

4. We will demonstrate that the proposed SLT code is capable of mitigating the effects of errors imposed by fast fading as well as of reducing the frequency of HARQ activation. However, in case of slow fading even the retransmitted SLT codewords might be corrupted owing to the presence of correlated fading. In this scenario the relay nodes are capable of providing a beneficial spatial diversity gain owing to their geographic separation. However, if the relay is far from the source, DF relaying may result in avalanche-like error propagation and in this scenario $\mathrm{AF}$ relaying constitutes a safer design option.

The outline of this paper is as follows. Section 2 summarises the characteristics of SLT codes using ID and its concatenation with the 16-QAM demodulator in order to iteratively exchange extrinsic information. The resultant coding scheme is referred to as the ID-SLT 16-QAM arrangement. In Section 3, the proposed coding scheme is applied to wireless relay networks in order to improve their performance. The system's architecture and mathematical characterisation is provided in Sections 4 and 5. Finally, Section 6 details our simulation results recorded for the HARQ-aided SLT-coded 16-QAM system, followed by our conclusions.

\section{Iterative decoding aided SLT codes}

\subsection{Iterative decoding-aided SLT codes}

LT codes [22] were originally designed for the binary erasure channel (BEC). When employed for transmission over wireless channels, which impose both fading and intersymbol interference (ISI), the LT code might become contaminated, which results in catastrophic inter-packet error propagation during LT decoding [23]. In order to mitigate the deleterious effects of error propagation, LT codes have been frequently combined with classic physicallayer FEC codes [23, 24]. The idea of combining classic FEC codes with LT codes by directly amalgamating them was proposed in [6], where systematically concatenated parity bits were incorporated in order to create the family of SLT codes.

The soft LT decoding process is based on the classic concept of low density parity check (LDPC) decoding. Given the generator matrix $\boldsymbol{G}$ of the LT code, we calculate the parity check matrix (PCM) $\boldsymbol{H}$ of the LT code similarly to that of a classic LDPC code, namely by partitioning the LT code's generator matrix into two matrices, where $\boldsymbol{A}$ and $\boldsymbol{B}$ have a size of $(K \times K)$ and $(K \times M)$, respectively. We choose the non-singular matrix $\boldsymbol{A}$ based on the conventional LT decoding process. Then the PCM is calculated as

$$
\boldsymbol{H}_{(M \times N)}=\left[\left(\boldsymbol{B}^{\mathrm{T}} \cdot\left(\boldsymbol{A}^{\mathrm{T}}\right)^{-1}\right)_{(M \times K)} \mid I_{(M \times M)}\right]
$$

where $\boldsymbol{A}^{\mathrm{T}}$ and $\boldsymbol{B}^{\mathrm{T}}$ is the transpose of A and B, respectively. An LT PCM can be represented by a classic Tanner graph [25]. To elaborate a little further, the filled circles and the filled squares of Fig. 1 represent the variable nodes and the check nodes of the LT code, respectively, while the horizontal lines connected to the variable nodes correspond to the intrinsic information provided by the channel's output. Let us assume that the circular node at the top of Fig. 1 represents the $k$ th variable node in the block of $N$ single-bit LT-encoded packets, which is also termed as the root node. The root node receives extrinsic parity-check information from the specific check nodes that are directly connected to it at the tree-level immediately below it, as seen in Fig. 1. Similarly, these check nodes also receive extrinsic information from the specific variable nodes they are directly connected to at the next level down in Fig. 1 etc. The dotted lines in Fig. 1 indicate that the above process is 


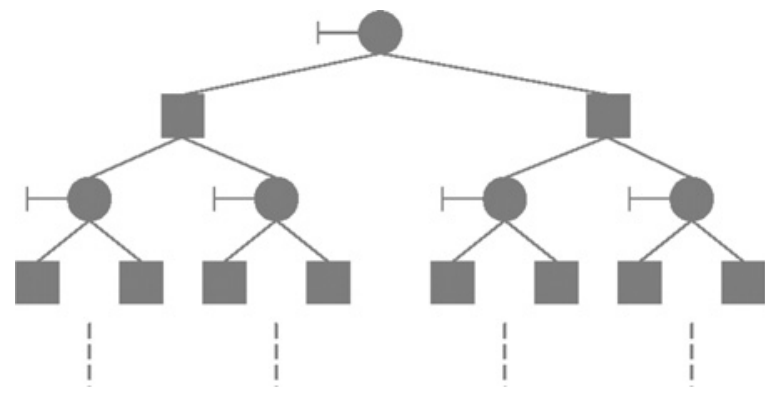

Fig. 1 Tree-based representation of LT codes

repeated further by expanding the tree. The number of connections associated with a variable node of the LT code - excluding the line representing the intrinsic information - indicates the column weight of this particular message node, while the number of connections associated with an LT check node represents the corresponding row weight. The column weight and row weight of the LT PCM are related to the degree distribution of LT packets. The LT decoding process is implemented in the same way as the classic LDPC decoding procedure. Initially, the LT decoder's soft values are set to a value corresponding to the demodulator's soft output. The decoder's soft values of $R_{i, j}^{a}$ and $Q_{i, j}^{a}$, which denote the log-likelihood ratio (LLRs) passed from the check nodes to the variable nodes and vice versa are then iteratively updated after each decoding iteration as follows

$$
\tanh \left(\frac{R_{i, j}}{2}\right)=\prod_{n \in\left\{C_{i}\right\}, n \neq i} \tanh \left(\frac{Q_{n, j}}{2}\right)
$$

The LT decoder outputs its tentative hard decision values after each iteration and then checks whether the product of the corresponding codeword and the transpose of the PCM $\boldsymbol{H}$ is equal to zero, that is whether a legitimate codeword was produced. If not, the LT decoding process will be continued in an iterative fashion, until the output codeword becomes legitimate or the maximum affordable number of iterations is exhausted.

In order to improve the achievable performance, the LT code's degree distribution is created by expanding its
$(K \times N)$-element generator matrix $\boldsymbol{A}$ with the aid of attaching a unity matrix having a size of $(K \times K)$, resulting in a systematic code [6]. Particularly, if we have a generator matrix $\boldsymbol{G}_{K \times N}=\left[I_{K \times K} \mid \boldsymbol{A}_{K \times M}\right]$, where $(N=K+M)$, then the PCM $\boldsymbol{H}$ is calculated as

$$
\boldsymbol{H}=\left[\boldsymbol{A}^{\mathrm{T}} \mid I^{\prime}\right]
$$

The original LT code of [22] utilised the robust soliton degree (RSD) distribution for bit encoding. However, the RSD distribution consists of many single-parity check bits, also known as degree-one nodes, which typically have a significantly lower reliability than information nodes. Hence, employing the RSD is not suitable for LT codes involving iterative soft decoding, especially when relying on simple parity check codes. To improve the achievable performance of both LT codes and SLT codes, the authors of $[23,26]$ have proposed several improved distributions, namely the improved RSD (IRSD) distribution and the truncated degree (TD) distribution. The TD distribution has a low number of degree-one nodes and hence will be employed for designing the proposed systems.

When utilising soft input and soft output (SISO) information for iterative decoding, the SLT decoder exchanges extrinsic information with the 16-QAM demodulator, which also uses SISO information in its bit-to-symbol demapping process, through a pair of interleaver and de-interleaver components. This model is shown in Fig. 2, where the soft output of the demodulator is fed forward to the SLT decoder. After carrying out SLT decoding, the resultant extrinsic information is fed back to the demodulator as the a priori information. The demodulator then exploits both the a priori information and the channel's output information, which is demapped to bits and passed to the SLT decoder again. This process continues, until the syndrome checking condition of the SLT decoder is satisfied or the affordable number of iterations is exhausted. The exchange of extrinsic information with the demodulator will enhance the attainable decoding performance of the SLT decoder. Consequently, a significant coding gain is achieved by the system. The performance of the iterative decoding process might be beneficially visualised by extrinsic information transfer (EXIT) charts [27].

Typically, the classic Gray bit-to-symbol mapping scheme is the best in non-iterative arrangements, but it does not
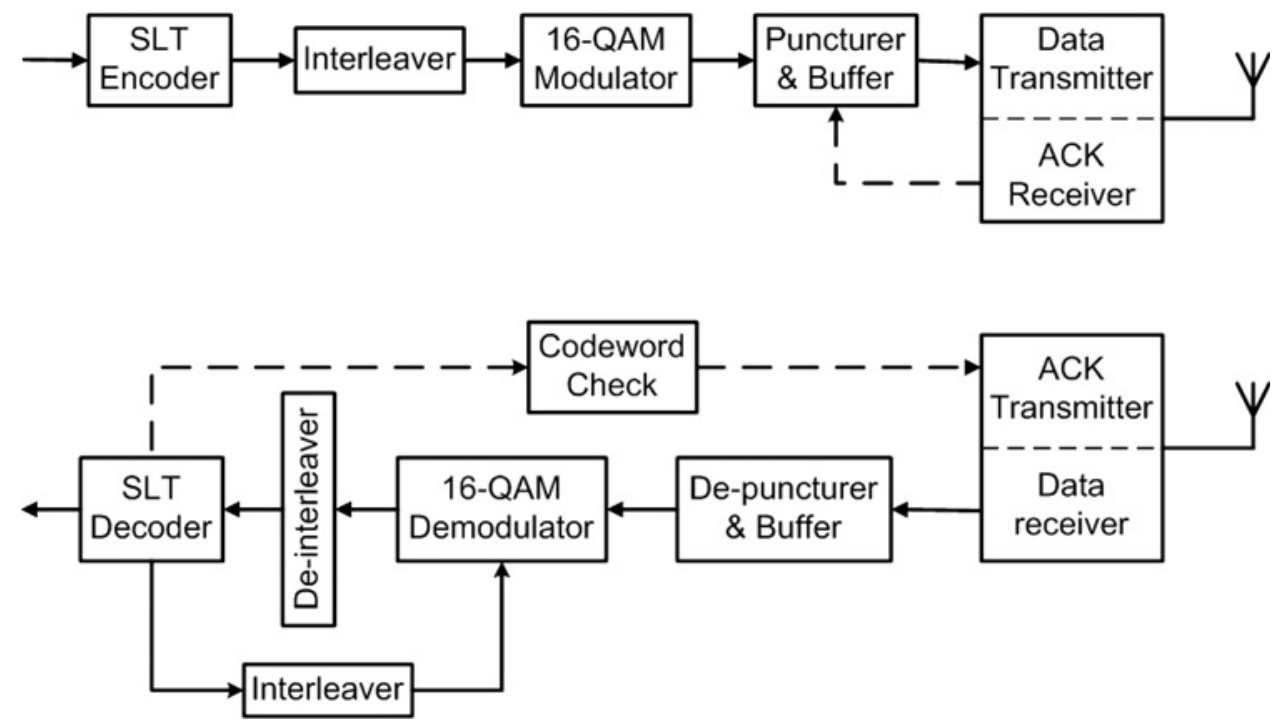

Fig. 2 Block diagram of the HARQ ID-SLT coded 16-QAM scheme 
benefit from iterative decoding [7]. Hence, in this contribution, a set-partitioning (SP)-based mapping scheme was chosen for the demodulator.

\subsection{EXIT chart}

As mentioned in the previous section, the SLT codes may be decoded by belief propagation. Hence, we can analyse SLT codes with the aid of EXIT charts [27]. According to [6], an EXIT chart may be formulated based on the LLR messages being passed between the SLT message nodes and parity check nodes. Let $Q^{\prime}$ and $R^{\prime}$ denote the LLR information passed from the message nodes to the check nodes and vice versa. The soft bit message corresponding to $Q^{\prime}$ is expressed as

$$
Q^{\prime}=\tanh \left(\frac{Q}{2}\right)
$$

The extrinsic LLR information passed from the check nodes to the message nodes is defined by

$$
Q^{\prime}=\sum_{i=0}^{d_{m}-1} R_{i}^{\prime}
$$

where $R_{0}^{\prime}=4 y / N_{0}$ with the channel's output being $y, R_{i}^{\prime}$ represents the LLR information followed from the check nodes to the message nodes, except from the specific check node generating the LLR information $Q^{\prime}$, and $d_{\mathrm{m}}$ is the degree of the message nodes.

The extrinsic LLR information $\mathrm{R}^{\prime}$ passed from the check nodes to the message nodes is defined as

$$
\tanh \left(\frac{R^{\prime}}{2}\right)=\prod_{i=1}^{d_{c}-1} \tanh \left(\frac{Q_{i}^{\prime}}{2}\right) \Leftrightarrow R=\prod_{i=1}^{d_{c}-1} R_{i}
$$

where $Q_{i}^{\prime}$ represents the LLR information gleaned from the message nodes, except from the specific message node generating the LLR information message $R^{\prime}$.

Let $m_{R}$ and $m_{Q}$ denote the mean of $R^{\prime}$ and $Q^{\prime}$. Then, from (5) we have

$$
m_{Q}^{(l)}=m_{R_{0}}+\left(d_{m}-1\right) m_{R}^{(l-1)}
$$

where $l$ is the iteration index and $m_{R_{0}}=4 E / N_{0}$, while $E$ is the transmit bit energy and $N_{0}$ is the noise power. Then, $m_{R}$ may be updated according to

$$
m_{R}^{(l)}=J^{-1}\left[I\left(X ; R^{(l)}\right]\right.
$$

where $J\left(m_{R}\right)$ is defined as

$$
\begin{aligned}
J\left(m_{R}\right)= & I\left(X ; R^{l}\right)=\int \frac{2}{\sqrt{4 \pi m_{R}}} \mathrm{e}^{-\left(\left(l-m_{R}\right) / 4 m_{R}\right)} \\
& \times\left[1-\log _{2}\left(1+\mathrm{e}^{-l}\right)\right] \mathrm{d} l
\end{aligned}
$$

Employing the mutual information equation in [28], (8) may be rewritten as

$$
m_{R}^{(l)}=J^{-1}\left(\frac{1}{\ln 2} \sum_{i=1}^{\infty} \frac{1}{2 i(2 i-1)}\left[\phi_{i}\left(m_{Q^{l}}\right)\right]^{d_{c}-1}\right)
$$

where

$$
\phi_{i}\left(m_{Q^{l}}\right)=\int_{-1}^{+1} \frac{2 t^{2 i}}{\left(1-t^{2}\right) \sqrt{4 \pi m_{Q^{l}}}} \mathrm{e}^{\left\{\left[\ln ((1+t) /(1-t))-m_{Q^{l}}\right]^{2} / 4 m_{Q}\right\}} \mathrm{d} t
$$

According to [6], the message node's EXIT function may be expressed as

$$
\begin{aligned}
I_{E_{m}} & =I\left(X, Q^{\prime}\right)=I\left(X ; R_{0}^{\prime}, R_{1}^{\prime}, \ldots, R_{d_{m}-1}^{\prime}\right) \\
& =f\left[I\left(X, R_{0}^{\prime}\right), I\left(X, R^{\prime}\right)\right]=f\left(I_{c h}, I_{A_{m}}\right) \\
& =J\left[J^{-1}\left\{I_{c h}+(j-1) J^{-1}\left(I_{A_{m}}\right)\right\}\right]
\end{aligned}
$$

and the check node's EXIT function is given by

$$
\begin{aligned}
I_{E_{c}} & =I\left(X, R^{\prime}\right)=I\left(X ; Q_{1}, \ldots, Q_{d_{c}-1}^{\prime}\right)=f\left[I\left(X, R^{\prime}\right)\right]=f\left(I_{A_{c}}\right) \\
& =\sum_{j=2}^{d_{c_{\max }}} d_{c}(j) \cdot \frac{1}{\ln 2} \sum_{i=1}^{\infty} \frac{1}{2 i(2 i-1)}\left[\phi_{i}\left\{J^{-1}\left(I_{A_{c}}\right)\right\}\right]^{j-1}
\end{aligned}
$$

Finally, the average extrinsic information at the output of the SLT decoder is expressed as

$$
I_{E_{\mathrm{SLT}}}=\left[I_{E_{m}} \mid I_{E_{c}}\right]
$$

As mentioned above, in the 16QAM-SLT receiver ID is carried out by exchanging extrinsic information between the 16-QAM demapper and the SLT decoder. Hence, as seen in Figs. 1 and 2 the output of the SLT decoder is a function of both the input mutual information of the demapper as well as of the output mutual information of the SLT decoder. Similarly, the output information of the demapper is a function of the information at the channel's output and of the SLT decoder's output mutual information.

\section{ID-SLT coding in wireless cooperative communications network}

A single-relay-node-aided cooperative communications scheme is proposed in this paper. The transmissions are divided into two time slots. During the first time slot, the source station broadcasts its data both to the RS and to the destination station (DS). In the second slot, the RS forwards data to the destination. Based on the two versions of the received signal, the DS recovers the original data.

\subsection{Path-loss-related power gain}

The path-loss plays an important role in the analysis and design of cooperation-aided systems, which may be modelled as [29]

$$
L_{a b}=\frac{K}{d_{a b}^{\alpha}}
$$

where $K$ is a constant depending on the propagation environment, $d_{a b}$ is the geometrical distance from node $a$ to node $b$ and $\alpha$ is the path-loss exponent. According to [30], we have $\alpha=2$ for the free-space model, while $\alpha=4$ for the ground reflection model. The relationship between the energy received at the RS $E_{\mathrm{sr}}$ and at the DS $E_{\mathrm{sd}}$ during the 
first time slot may be expressed as

$$
E_{\mathrm{sr}}=\frac{L_{\mathrm{sr}}}{L_{\mathrm{sd}}} E_{\mathrm{sd}}=G_{\mathrm{sr}} E_{\mathrm{sd}}
$$

where $G_{\text {sr }}$ may be referred to as the path-loss-related power gain for the source-to-relay (SR) link. When taking into account (15), $G_{\text {sr }}$ is equal to

$$
G_{\mathrm{sr}}=\left(\frac{d_{\mathrm{sd}}}{d_{\mathrm{sr}}}\right)^{\alpha}
$$

Similarly, the path-loss-related power gain for the relay-todestination (RD) link is expressed as

$$
G_{\mathrm{rd}}=\left(\frac{d_{\mathrm{sd}}}{d_{\mathrm{rd}}}\right)^{\alpha}
$$

In the next two subsections, $\mathrm{AF}$ and $\mathrm{DF}$ relaying will be detailed.

\subsection{AF scheme}

During the first time slot, the source broadcasts its signal $x_{s, k}$. The signals received at the RS and the DS, namely $y_{s r, k}$ and $y_{s d, k}$ are expressed as

$$
\begin{gathered}
y_{\mathrm{sr}, k}=\sqrt{G_{\mathrm{sr}}} \sqrt{P_{s}} h_{\mathrm{sr}, k} x_{s, k}+n_{\mathrm{sr}, k} \\
y_{\mathrm{sd}, k}=\sqrt{G_{\mathrm{sd}}} \sqrt{P_{s}} h_{\mathrm{sd}, k} x_{s, k}+n_{\mathrm{sd}, k}
\end{gathered}
$$

where $k \in\left\{1,2, \ldots, N_{s}\right\}$ is symbol index, $N_{\mathrm{s}}$ is the number of transmitted symbols, $h_{\mathrm{sr}, k}$ and $h_{\mathrm{sd}, k}$ are the complex nondispersive Rayleigh fading factors, while $n_{\mathrm{sr}, k}$ and $n_{\mathrm{sd}, k}$ represent the additive white Gaussian noise (AWGN) with zero mean and a variance of $N_{0}$.

The signal received from the source by the RS is amplified and forwarded to the destination. The AF gain $\beta$ at the RS is chosen as [10]

$$
\beta \leq \sqrt{\frac{1}{G_{\mathrm{sr}} P_{s}\left|h_{\mathrm{sr}}\right|^{2}+N_{0}}}
$$

Naturally, the unwanted noise and interference are also amplified by the RS and forwarded to the DS. The relayed signal arriving at the DS is expressed as

$$
y_{\mathrm{rd}, k}=\sqrt{G_{\mathrm{rd}}} \sqrt{P_{\text {relay }}} h_{\mathrm{rd}, k} \beta y_{\mathrm{sr}, k}+n_{\mathrm{rd}, k}
$$

At the DS, the receiver combines the two received signal versions $y_{\text {sd }}$ and $y_{\text {rd }}$ into

$$
y_{d, k}=y_{\mathrm{sd}, k}+y_{\mathrm{rd}, k}
$$

and then passes them to the demodulator. Finally, SLT decoding is carried out, as detailed in the previous section. Having briefly considered AF relaying, let us now discuss DF relaying.

\subsection{DF scheme}

The signal processing operations carried out during the first time slot are identical to those of the AF scheme. The RS's receiver, however, decodes the received signal and re-encodes them, before forwarding them to the destination. Hence, (10) has to be replaced by

$$
y_{\mathrm{rd}, k}=\sqrt{G_{\mathrm{rd}}} \sqrt{P_{\text {relay }}} h_{\text {rd }, k} x_{\text {relay }, k}+n_{\mathrm{rd}, k}
$$

where $x_{\text {relay }, k}$ is the re-encoded signal at the RS.

The decoding process at the destination ensues similar to that of the AF scheme.

\section{HARQ with incremental redundancy for ID-SLT coding}

Again, there are two basic types of HARQ, namely the Type-I HARQ and the Type-II HARQ. The transmitter of the classic Type-I HARQ scheme typically retransmits all the information and parity bits of corrupted packets, when a negative acknowledgement (ACK) is received, while the receiver simply drops erroneous packets [31]. In the H-ARQ type II scheme, the information part and the parity part are sent together during the first transmission attempt. However, during the second transmission attempt additional parity information is transmitted. There are also two ways of information combining in H-ARQ protocol, namely chase combining and incremental redundancy (IR-HARQ). When the different received replicas are combined in the soft-value domain, the resultant technique is referred to as the HARQ using chase combining. In fact, the method simply boosts the received SNR after chase combining. In other words, it does not provide an additional coding gain and does not increase the achievable effective throughput. By contrast, the Type-II HARQ uses incremental redundancy (IR-HARQ) where the transmitter sends additional redundancy during the retransmission stages. The receiver combines the additional redundant bits with those received before, in order to recover the original information bits. Naturally, this method is expected to provide an increased coding gain at the receiver. Owing to these advantages, in this section the SLT-coded HARQ using IR is chosen for investigation here.

The IR-HARQ-aided SLT coding scheme is shown in Fig. 2. As shown in this figure, the information bits are encoded and modulated, before they are passed to the puncturing and buffering components. A fraction of the parity bits is punctured, in order to achieve the highest possible code rate during the first transmission, while storing the remaining punctured bits for IR transmissions, as and when needed. In the receiver, the SLT decoder and the syndrome checking block will generate a status signal in order to inform the transmitter about the outcome of the CRC check operation. If a positive ACK is received corresponding to a legitimate decoded code word, the buffered parity bits that were previously punctured will be deleted. Otherwise, incremental redundancy is transmitted, until we reach the maximum affordable number of IR transmissions. In this paper, the original cooperative IR-HARQ scheme is referred to as the 'passively-cooperative IR-HARQ'.

In this contribution, an 'actively-cooperative IR-HARQ' scheme is proposed for cooperative wireless networks. There, both the relay's and the destination's receivers feed their decoding status back to the source. If negative ACKs are received from both the RS and the DS, the IR transmission is carried out as usual. If a negative ACK is received from the DS and a positive ACK is received from the RS, the IR transmissions are implemented at the relay only, while the source remains idle. Clearly, the 
arrangement requires the collaboration among all stations, especially the assistance of the RS. Note that this IR-HARQ procedure is only suitable for the DF cooperative scheme and the IR transmissions require extra timeslots.

During IR transmission in the actively cooperative IRHARQ scheme, only a single version of the signal may have arrived from the RS to the DS. Thus, there may not be any spatial diversity gain. To compensate for the resultant diversity loss, the relay may choose to increase its transmit power. This power allocation may be deemed fair, because the source has not utilised its power during the IR transmissions, provided of course that the total transmit power remains constant. The associated power consumption will be quantified in the next section.

The actively cooperative scheme here can be seen as an instance of the HARBINGER protocol [32]. However, the scheme does not always prove to be more effective than the passive one. Hence, we will investigate the conditions to apply the actively cooperative scheme in the following section.

\section{Capacity and power}

The achievable capacity and power requirements of both the passively and actively cooperative IR-HARQ schemes are quantified in this section. Based on these quantitative results, we will propose measures to assist the 'actively cooperative' scheme in performing more efficiently at a lower transmit power than the 'passively cooperative' one. For a convenient analysis, in the actively cooperative IRHARQ scheme, the IR transmissions are divided into two time slots as well. During the first time slot, the RS's transmit power is set to $P_{\text {relay }}^{\prime}$, while in the second slot, it is set to $P_{\text {relay }}$.

Let us assume that during the first transmission of the passively cooperative scheme, the source sends $m_{c}$ bits and in each IR transmission, it sends $l_{c}$ bits, while the number of IR transmissions is $n \in\left\{1,2, \ldots, n_{\max }\right\}$, where $n_{\max }$ is the maximum number of IR transmissions. For the actively cooperative scheme, the first transmission is the same as that of the passive cooperative scheme. The number of IR transmissions at both the source and the RS is denoted as $n_{s}$, while the number of IR transmissions at the RS only is $n_{r}$. Also note that we have to obey $n_{s}+n_{r}=n$ to make the comparison between the two systems a fair one.

\subsection{Capacity}

1. Passively-cooperative scheme: According to Proposition 2 of [33], the instantaneous capacity of the first transmission of a passively cooperative single-relay-aided IR-HARQ system in fading channels may be expressed as

$$
C_{1^{s t}, \text { passive }}=\min \left\{C_{1^{s t}, \text { passive }}^{1}, C_{1^{s t}, \text { passive }}^{2}\right\}
$$

with

$$
\begin{aligned}
C_{1^{s t}, \text { passive }}^{1} & =\frac{1}{2} \log _{2}\left(1+\frac{P_{s}\left|h_{\mathrm{sr}}\right|^{2}}{d_{\mathrm{sr}}^{\alpha} N_{0}}\right) \\
C_{1^{s t}, \text { passive }}^{2}= & \frac{1}{2} \log _{2}\left(1+\frac{P_{s}\left|h_{\mathrm{sd}}\right|^{2}}{d_{\mathrm{sd}}^{\alpha} N_{0}}\right) \\
& +\frac{1}{2} \log _{2}\left(1+\frac{P_{\text {relay }}\left|h_{\mathrm{rd}}\right|^{2}}{d_{\mathrm{rd}}^{\alpha} N_{0}}\right)
\end{aligned}
$$

Averaging (26) and (27) over the probability density function of the Rayleigh distribution and applying the same processes as in [34], the average capacity of the first transmission becomes

$$
\bar{C}_{1^{s t}, \text { passive }}=\min \left\{f\left(\frac{P_{s}}{d_{\mathrm{sr}}^{\alpha} N_{0}}\right), \quad f\left(\frac{P_{s}}{d_{\mathrm{sd}}^{\alpha} N_{0}}\right)+f\left(\frac{P_{\text {relay }}}{d_{\mathrm{rd}}^{\alpha} N_{0}}\right)\right\}
$$

where we have [34]

$$
f(x)=-\log _{2} \mathrm{e} \cdot \mathrm{e}^{-1 / x} E i\left(\frac{-1}{x}\right)
$$

with $\operatorname{Ei}(y)$ being the exponential-integral function [34].

Similarly, the average capacity of each IR transmission of a passively cooperative single-relay-aided IR-HARQ system in fading channels is given by

$$
\bar{C}_{I R, \text { passive }}=\min \left\{f\left(\frac{P_{s}}{d_{\mathrm{sr}}^{\alpha} N_{0}}\right), \quad f\left(\frac{P_{s}}{d_{\mathrm{sd}}^{\alpha} N_{0}}\right)+f\left(\frac{P_{\text {relay }}}{d_{\mathrm{rd}}^{\alpha} N_{0}}\right)\right\}
$$

Hence, the average capacity of a passively cooperative singlerelay-aided IR-HARQ system in fading channels may be expressed as

$$
\begin{aligned}
\bar{C}_{\text {passive }}= & \frac{m_{c}}{m_{c}+n l_{c}} \bar{C}_{1^{s t} \text {,passive }}+\frac{n l_{c}}{m_{c}+n l_{c}} \bar{C}_{I R, \text { passive }} \\
= & \frac{m_{c}}{m_{c}+n l_{c}} \bar{C}_{1^{s t} \text {,passive }}+\frac{n_{s} l_{c}}{m_{c}+n l_{c}} \bar{C}_{I R, \text { passive }} \\
& +\frac{n_{r} l_{c}}{m_{c}+n l_{c}} \bar{C}_{I R, \text { passive }}
\end{aligned}
$$

2. Actively cooperative scheme: When the relay decoded a packet correctly and actively transmitted the request IR information only, the system's instantaneous capacity is given by

$$
\begin{aligned}
C_{I R, \text { active }}= & \frac{1}{2} \log _{2}\left(1+\frac{P_{\text {relay }}^{\prime}\left|h_{\text {rd }}^{\prime}\right|^{2}}{d_{\text {rd }}^{\alpha} N_{0}}\right) \\
& +\frac{1}{2} \log _{2}\left(1+\frac{P_{\text {relay }}\left|h_{\text {rd }}\right|^{2}}{d_{\text {rd }}^{\alpha} N_{0}}\right)
\end{aligned}
$$

Subsequently, its average capacity during the active IR transmission is expressed as

$$
\bar{C}_{I R, \text { active }}=f\left(\frac{P_{\text {relay }}^{\prime}}{d_{\text {rd }}^{\alpha} N_{0}}\right)+f\left(\frac{P_{\text {relay }}}{d_{\mathrm{rd}}^{\alpha} N_{0}}\right)
$$

Noted that the source stop sending IR information only, when the decoding process at the relay is successful. Therefore the average capacity of an actively cooperative single-relay-aided 
IR-HARQ system in fading channels may be expressed as

$$
\begin{aligned}
\bar{C}_{\text {active }}= & \frac{m_{c}}{m_{c}+n l_{c}} \bar{C}_{1^{s t} \text {,passive }}+\frac{n_{s} l_{c}}{m_{c}+n l_{c}} \bar{C}_{I R, \text { passive }} \\
& +\frac{n_{r} l_{c}}{m_{c}+n l_{c}} \bar{C}_{I R \text {,active }}
\end{aligned}
$$

3. Comparison: The difference between (31) and (34) manifests itself in the third term. Therefore the capacity of the actively cooperative scheme is higher than or equal to that of the passively cooperative one, if $\bar{C}_{\text {active }} \geq \bar{C}_{\text {passive. }}$. In other words, the relay's allocated power and its position have to satisfy the following condition

$$
\begin{aligned}
& \left\{f\left(\frac{P_{\text {relay }}^{\prime}}{d_{\text {rd }}^{\alpha} N_{0}}\right)+f\left(\frac{P_{\text {relay }}}{d_{\text {rd }}^{\alpha} N_{0}}\right)\right\} \\
& \quad \geq \min \left\{f\left(\frac{P_{s}}{d_{\mathrm{sr}}^{\alpha} N_{0}}\right), f\left(\frac{P_{s}}{d_{\mathrm{sd}}^{\alpha} N_{0}}\right)+f\left(\frac{P_{\text {relay }}}{d_{\mathrm{rd}}^{\alpha} N_{0}}\right)\right\}
\end{aligned}
$$

\subsection{Transmit power}

The average total transmit energy of each symbol of the passive cooperative single-relay-aided IR-HARQ system is expressed as

$$
\begin{aligned}
\bar{P}_{\text {passive }} & =P_{s}+P_{\text {relay }}=\frac{\left(m_{c}+n l_{c}\right)\left(P_{s}+P_{\text {relay }}\right)}{m_{c}+n l_{c}} \\
& =\frac{\left(m_{c}+n_{s} l_{c}\right)\left(P_{s}+P_{\text {relay }}\right)}{m_{c}+n l_{c}}+\frac{n_{r} l_{c}\left(P_{s}+P_{\text {relay }}\right)}{m_{c}+n l_{c}}
\end{aligned}
$$

while that of the actively cooperative single-relay-aided IRHAQR system is calculated by replacing $P_{s}$ in the second term of (36) by $P_{\text {relay }}^{\prime}$, yielding

$$
\bar{P}_{\text {active }}=\frac{\left(m_{c}+n_{s} l_{c}\right)\left(P_{s}+P_{\text {relay }}\right)}{m_{c}+n l_{c}}+\frac{n_{r} l_{c}\left(P_{\text {relay }}^{\prime}+P_{\text {relay }}\right)}{m_{c}+n l_{c}}
$$

It may be inferred from (36) and (37) that $\bar{P}_{\text {passive }} \geq \bar{P}_{\text {active }}$ if the following condition is satisfied

$$
P_{\text {relay }}^{\prime} \leq P_{S}
$$

Based on the analysis provided above, it is concluded that both inequalities (35) and (38) must be satisfied to allow the 'actively cooperative scheme' achieve a higher capacity with less transmit power, compared to the 'passively cooperative scheme', if the three stations collaborate.

The channel capacity of the passively and actively cooperative schemes is shown in Fig. 3, where we have $P_{s}=0.67, \quad P_{\text {relay }}=0.33, \quad P_{\text {relay }}^{\prime}=0.33$ and $d_{\mathrm{sr}}=d_{\mathrm{rd}}=$ $0.5 d_{\text {sd }}$. Clearly, the actively cooperative scheme may achieve equal or higher capacity than the passively cooperative one. This fact means that the relay's position and power arrangement in the actively cooperative scenario indeed satisfied the required conditions.

Both these schemes impose, however, a factor two multiplexing loss because of splitting a time slot into two phases: broadcasting and relaying, as described in Section 4. To recover the multiplexing loss, successive relaying was employed in $[35,36]$, which may also be applied to our scheme.

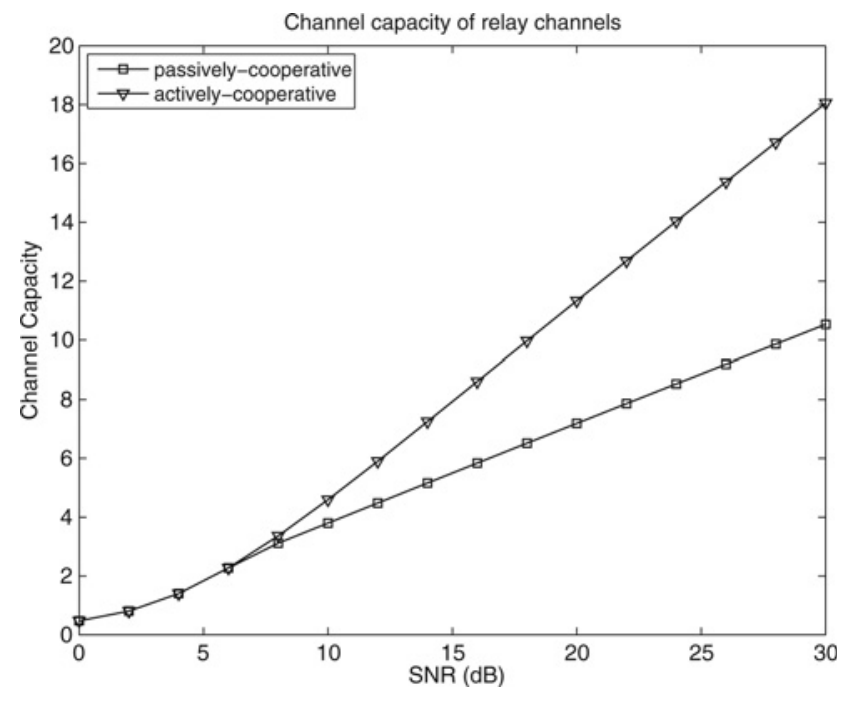

Fig. 3 Channel capacity of cooperative schemes communicating in uncorrelated Rayleigh fading channels: $P_{s}=0.67, P_{\text {relay }}=0.33$, $P_{\text {relay }}^{\prime}=0.67$, and $d_{s r}=d_{r d}=0.5 d_{s d}$

\section{System performance}

The BER performance of the ID-SLT-coded 16QAM scheme of Fig. 2 is shown in Fig. 4. The half-rate SLT code $\{1200$, $2400\}$ is used for all three investigated schemes, including the direct transmission, AF and DF arrangements. The detailed parameters of each scheme can be seen in Table 1. It is observed in Fig. 4 that there is an approximately $2.5 \mathrm{~dB}$ gain, when the 16-QAM mapper and the SLT decoder iteratively exchange their extrinsic information in the context Scheme-1 of Table 1. Introducing AF relaying in Scheme-2 provides only a slightly improvement of about $0.2 \mathrm{~dB}$. As mentioned before, AF relaying simply amplifies the received signal at the relay and then forwards it. Thus, it does not benefit from the coding gain of SLT coding. A clearer view is provided by the EXIT chart [7] of Fig. 5. There is only a tiny gap between the EXIT functions of

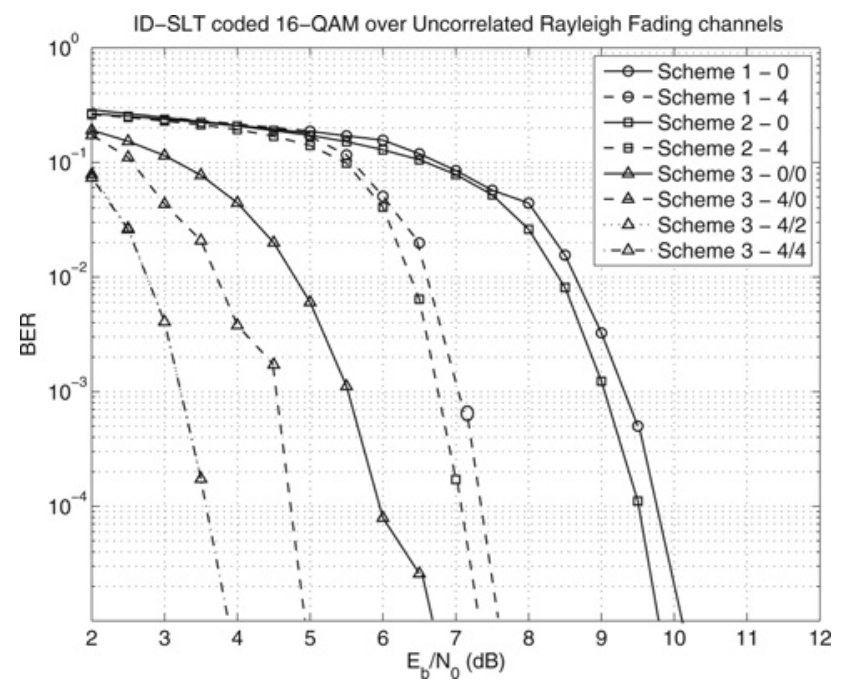

Fig. 4 BER of the ID-SLT-coded 16-QAM schemes as shown in Table 1

Scheme A - x: Scheme A uses $\mathrm{x}$ inner iterations between SLT decoder and 16-QAM demapper

Scheme B - y/z: Scheme B uses y inner iterations between SLT decoder and 16-QAM demapper at the DS; and uses $\mathrm{z}$ inner iterations between SLT decoder and 16-QAM demapper at the RS 


\section{www.ietdl.org}

Table 1 ID-SLT-coded 16-QAM schemes' parameters

\begin{tabular}{lccc}
\hline Parameters & Scheme-1 & Scheme-2 & Scheme-3 \\
\hline relaying type & none & AF & DF \\
No. of data bits & 1200 & 1200 & 1200 \\
SLT code rate & $1 / 2$ & $1 / 2$ & $1 / 2$ \\
degree distribution type & TDD & TDD & TDD \\
modulation type & $16-$ OAM & $16-$ OAM & $16-$ OAM \\
No. of outer iter. & 8 & 8 & 8 \\
No. of inner iter. at RS & unavailable & unavailable & 0,2 and 4 \\
No. of inner iter. at DS & 0 and 4 & 0 and 4 & 0 and 4 \\
Rayleigh fading type & uncorrelated uncorrelated uncorrelated
\end{tabular}

Outer iter.: iterations inside SLT decoders; inner iter.: iterations between SLT decoders and 16-OAM demodulator

EXIT chart of ID-SLT coded 16QAM at $\mathrm{E}_{\mathrm{b}} / \mathrm{N}_{0}=4 \mathrm{~dB} \& 5 \mathrm{~dB}$

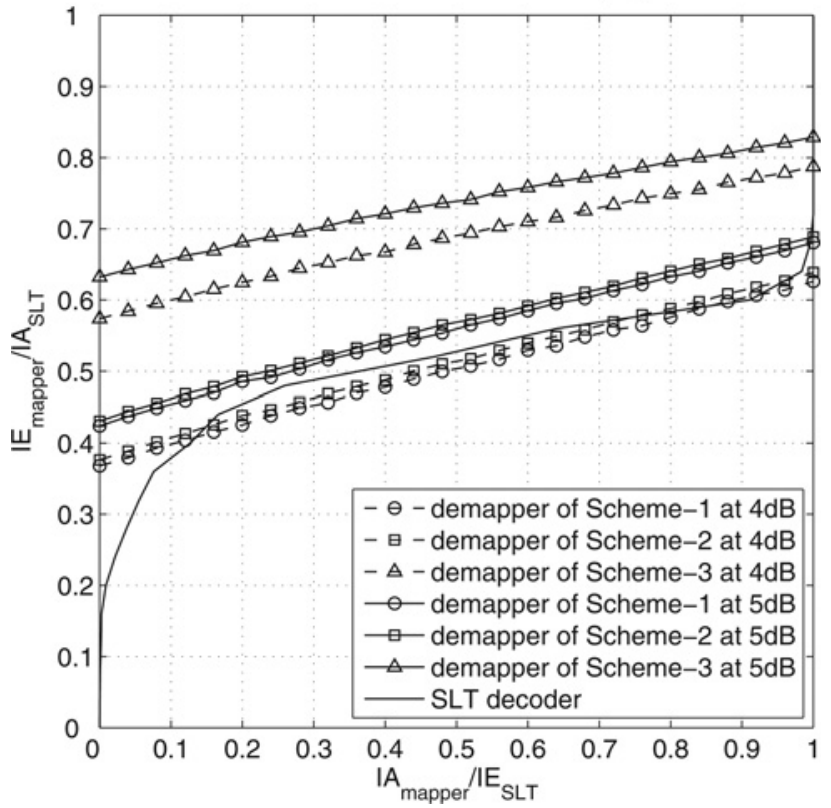

Fig. 5 EXIT chart for the ID-SLT-coded 16-QAM schemes over uncorrelated Rayleigh fading channels at $E_{b} / N_{0}=4$ and $5 \mathrm{~dB}$

Scheme-1 and Scheme- 2 of Table 1, which explains why AF relaying provides only insignificant benefits for the ID-SLTcoded 16-QAM system. By contrast, Scheme-3 of Table 1 first recovers and then re-encodes the source's bits at the relay. Thus, this process benefits from the coding gain of the SLT code. Therefore its performance becomes substantially better than that of the two previous schemes. More particularly, a $3 \mathrm{~dB}$ gain was achieved for the same system even without inner iterations between the SLT decoder and the 16-QAM symbol-to-bit demapper. There is a further gain of approximately $2.5 \mathrm{~dB}$, when four inner iterations are applied at the relay. Further insights are provided by Fig. 5. In this figure, the EXIT chart reveals that at $E_{b} / N_{0}=4 \mathrm{~dB}$ there is a widely open EXIT-tunnel for Scheme-3, which is not the case for Scheme-1 and Scheme-2. This implies that the DF relaying scheme, namely Scheme- 3 of Table 1 , is expected to outperform the two remaining schemes.

Although not explicitly shown here, we found that changing the number of inner decoding iterations at the relay also affects the signal quality at the destination. When increasing the number of inner iterations from one to two without increasing the number of inner iterations at the destination's receiver, the required SNR is reduced by about $1 \mathrm{~dB}$. However, no additional obvious improvements are attained, when the number of iterations is further increased, as evidenced by the two overlapped curves of Scheme 3 in Fig. 4. This fact may be explained by the EXIT chart of Fig. 6, where an open tunnel does exist, when there are two or four inner iterations at the relay, while it does not appear for a single iteration. Explicitly, an open tunnel emerges at $2 \mathrm{~dB}$ for two or more iterations. However, the difference between the EXIT functions of two and four iterations is extremely small. This is why the performance does not

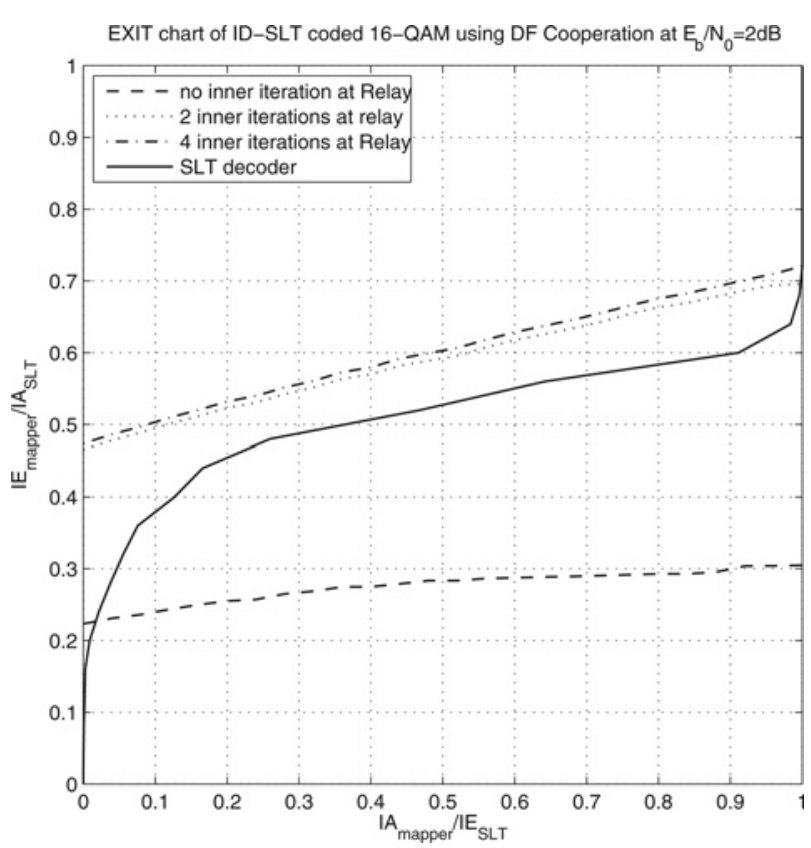

Fig. 6 EXIT chart for the ID-SLT-coded 16-QAM schemes using $D F$ cooperation (Scheme-3) over uncorrelated Rayleigh fading channels at $E_{b} / N_{0}=2 d B$

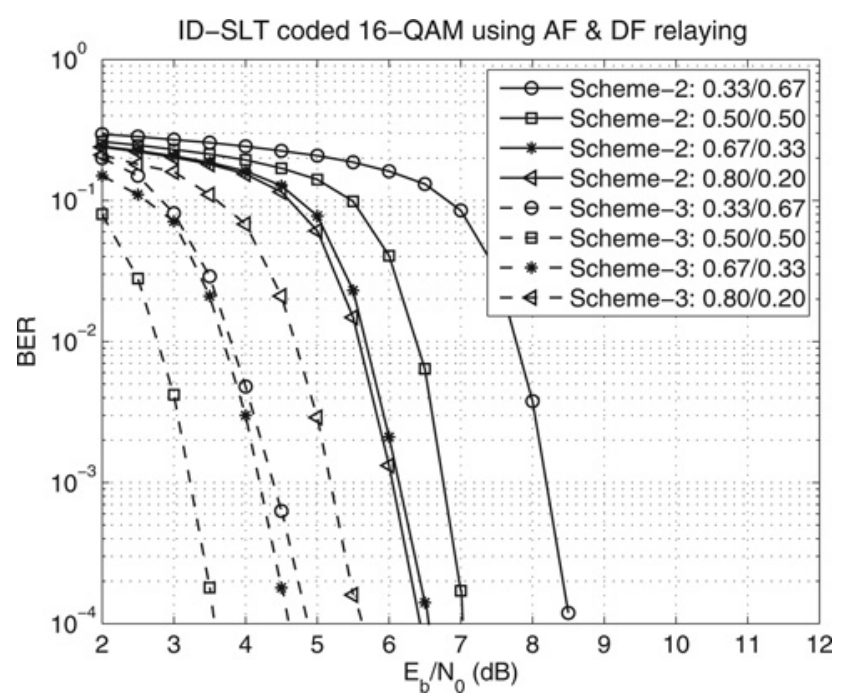

Fig. 7 BER of the ID-SLT-coded 16-QAM scheme over uncorrelated Rayleigh fading channels in conjunction with $A F$ and $D F$ cooperation using different normalised source powers: $P_{s}=\left\{\begin{array}{llll}0.33,0.50, & 0.67,0.80\end{array}\right\}$ and $P_{\text {relay }}=1-P_{s} ;$ normalised distance of $d_{s r}=0.5$ and $d_{r d}=1-d_{s r}$ 
substantially improve, when there are four iterations between the demapper and SLT decoder at the relay.

Fig. 7 illustrates our BER results for Scheme-2 and Scheme- 3 of Table 1 at different source powers $P_{s}$. We assumed that the total transmit power was fixed to unity, and the position of the RS is fixed right at the mid-point between the source and the DS. In Scheme-2, the BER degrades, when the sources' power is increased. An $E_{b} / N_{0}$ difference of $2 \mathrm{~dB}$ emerges, when increasing the sources' power from 0.33 to 0.80 . However, the system performs best at $P_{s}=0.5$ in the context of Scheme-3. Both increasing and decreasing the sources' power will degrade the BER.

In Fig. 8, we assumed that the transmit powers at both the source and relay were 0.5 , and the normalised distance between the source and the destination is unity. It can be observed in the figure that Scheme-2 performs slightly better, when the relay gets closer to the source, that is, for $d_{\text {sr }}=0.33$. Explicitly, an $E_{b} / N_{0}$ improvement of $0.5 \mathrm{~dB}$ is seen, when changing the source-to-relay distance from 0.67 to 0.33 . This observation suggests that Scheme-2 performs better for a relatively high received signal quality than for a lower quality, because in the latter case, it may suffer from excessive noise amplification. By contrast, as observed in Fig. 8, the best location for Scheme-3 appears at $d_{\mathrm{sr}}=0.5$, where relaying achieves a good balance between correcting errors and avoiding error propagation.

The following part provided simulation results for IRHARQ-aided SLT coding in our wireless relay network. In these simulations, the SLT code $\{1000,3000\}$, encoding 1000 uncoded bits into 3000 coded bits, was chosen, while the other parameters were provided in Table 2. The first transmission employs the SLT code $\{1000,2000\}$, and each IR transmission includes 200 extra bits. The maximum number of IR transmissions is five, which hence corresponds to the SLT code $\{1000,3000\}$. The source's and relay's transmit powers are 0.67 and 0.33 , respectively. If an IR transmission is required and the codeword recovered at the relay is legitimate, the relay will forward the data at a power of $P_{\text {relay }}^{\prime}=P_{s}$. It is also noted that these parameters allow the system to satisfy the conditions outlined in Section 5.

Fig. 9 showed the frame error ratio (FER) for the three scenarios listed in Table 2. According to Fig. 9, the performances of the actively and passively cooperative schemes are comparable, while they both outperformed the classic HARQ scheme dispensing with relaying. More particularly, observe in Fig. 9 that the power gain of more

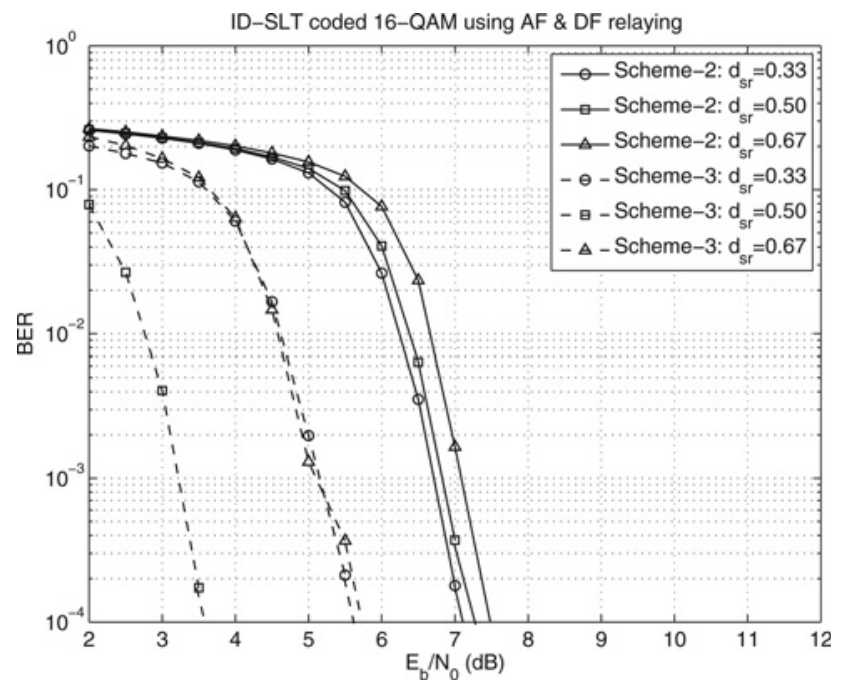

Fig. 8 BER of the ID-SLT-coded modulation scheme over uncorrelated Rayleigh fading channels in conjunction with AF and $D F$ cooperation using normalised source powers: $P_{s}=0.50$ and $P_{\text {relay }}=1-P_{s}$; different normalised distance: $d_{s r}=\{0.33$, $0.50,0.67\}$ and $d_{r d}=1-d_{s r}$

than $3 \mathrm{~dB}$ may be achieved in the cooperative schemes, compared to direct HARQ transmissions. The number of IR transmissions for these schemes is provided in Fig. 10. Despite sending an equal number of IR transmissions from the relay, the actively cooperative Scheme-C of Table 2 is capable of significantly reducing the number of IR transmissions at the source in the low $E_{b} / N_{0}$ region, namely below $4 \mathrm{~dB}$. Consequently, the actively cooperative scheme improves both the throughput and transmit power efficiency for the entire system.

The throughput of the three schemes of Table 2 is characterised in Fig. 11 as a direct consequence of Figs. 9 and 10. The throughput of Scheme-B and Scheme-C of Table 2 is significantly increased in the $E_{b} / N_{0}$ region between 3 and $7 \mathrm{~dB}$, compared to Scheme-A, where no cooperation was employed. Furthermore, Scheme-C may offer a slightly higher throughput than Scheme-B, despite its lower power consumption. The total transmit power requirements are revealed in Fig. 12. In accordance with the number of IR transmission at the source, Scheme-C is capable of reducing the total transmit power by nearly $5 \%$ in the $E_{b} / N_{0}$ region between 2 and $3.5 \mathrm{~dB}$, before it

Table 2 HARO/ID-SLT-coded 16-OAM schemes' parameters

\begin{tabular}{lccc}
\hline Parameters & Scheme-A & Scheme-B & Scheme-C \\
\hline Relaying type & none & passive DF & active DF \\
No. of data bits & 1000 & 1000 & 1000 \\
SLT code rate & $1 / 3$ & $1 / 3$ & $1 / 3$ \\
No. of bits of $1^{\text {st }}$ transmission & 2000 & 2000 & 2000 \\
maximum no. of IR transmissions & 5 & 5 & 5 \\
No. of bits per IR transmission & 200 & 200 & 200 \\
degree distribution type & TDD & $16-$ OAM & TDD \\
modulation type & $16-$ OAM & 8 & $16-$ QAM \\
No. of outer iter. & 8 & 4 & 8 \\
No. of inner iter. at RS & unavailable & 4 & 4 \\
No. of inner iter. at DS & 4 & uncorrelated & 4 \\
Rayleigh fading type & uncorrelated & uncorrelated \\
\hline
\end{tabular}




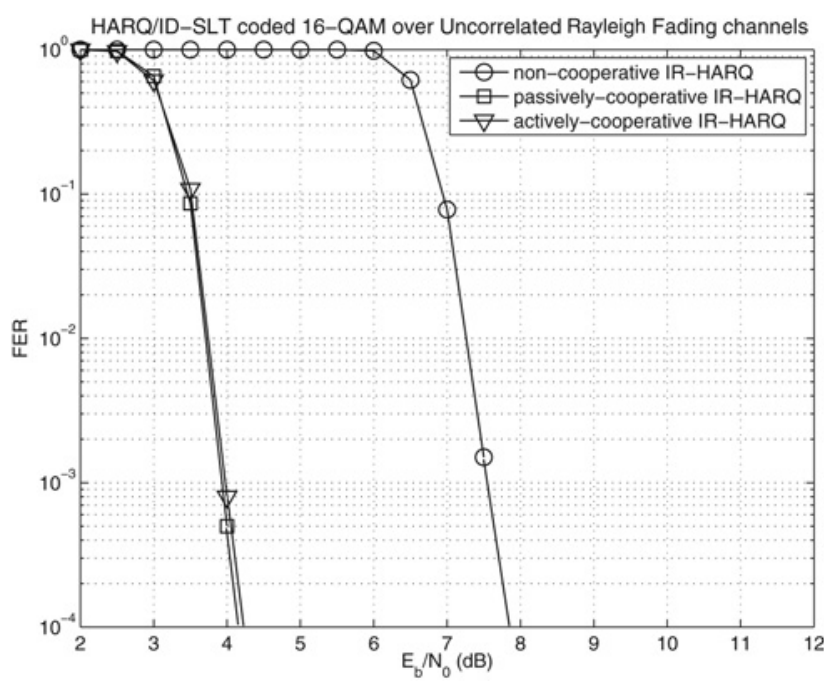

Fig. 9 FER of the ID-SLT-coded 16-QAM schemes as shown in Table 2

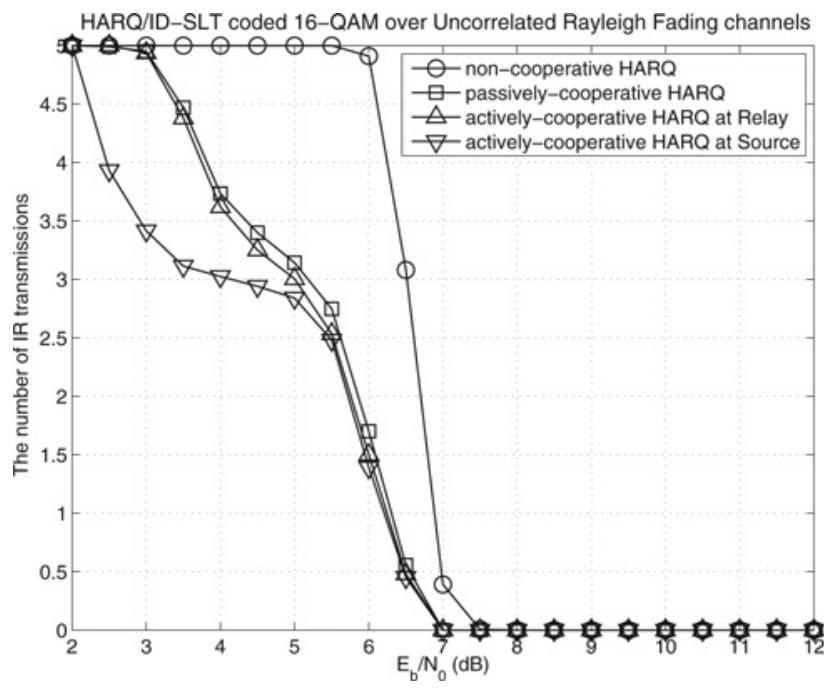

Fig. 10 Number IR transmissions of the ID-SLT-coded 16-QAM schemes as shown in Table 2

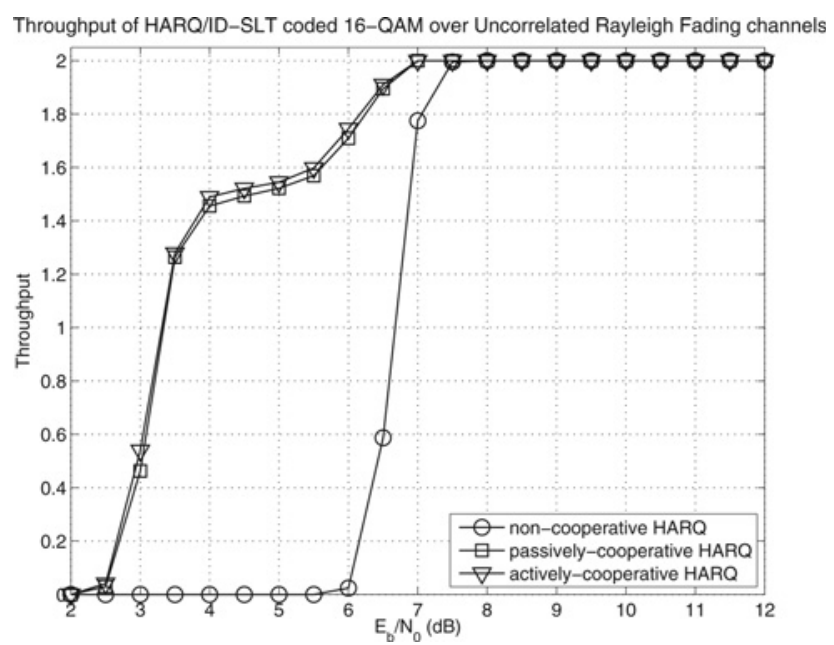

Fig. 11 Throughput of the ID-SLT-coded 16-QAM schemes as shown in Table 2

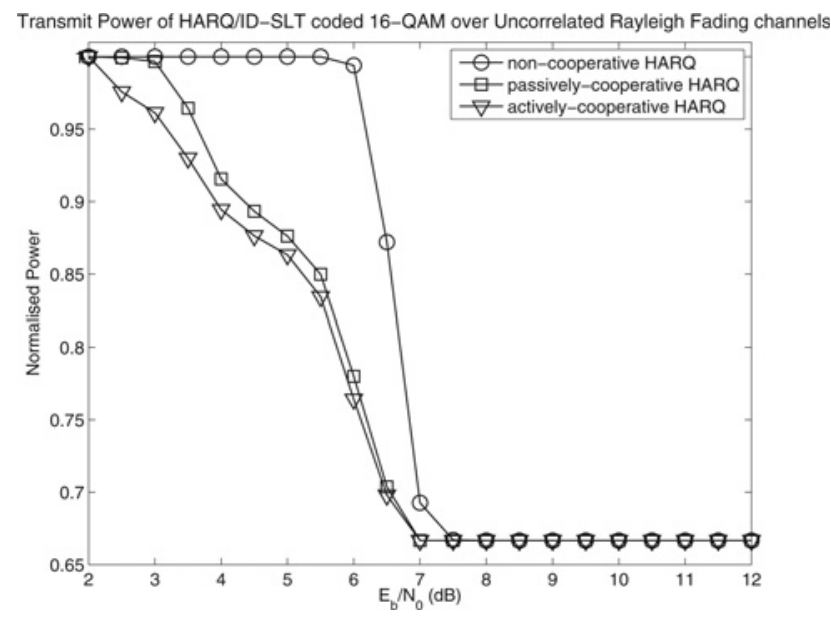

Fig. 12 Average transmit energy of the ID-SLT-coded 16-QAM schemes as shown in Table 2

gradually decreases and finally approaches that of Scheme-C around $6.5 \mathrm{~dB}$.

\section{Conclusions}

In this paper, we have proposed a HARQ aided SLT coded 16-QAM system for wireless relay-aided networks in order to improve the attainable system performance. The simulation results show that when $\mathrm{AF}$ and DF relaying were employed along with the proposed SLT coded 16-QAM scheme, the system achieved a gain of about 2.5 and $6 \mathrm{~dB}$, respectively, at the BER of $10^{-5}$, compared to the non-ID scheme operating without the relay's assistance. Moreover, we found that the AF relaying-aided SLT-coded 16-QAM scheme benefits more substantially when the RS is roaming close to the source. By contrast, the DF relaying-aided scheme achieves its best performance near the mid-point. The system's performance was improved by the actively cooperative ARQ protocol combined with SLT coding. More particularly, it reduces the number of IR transmissions by approximately $30 \%$, while the total transmit power was reduced by about $5 \%$ in the $E_{b} / N_{0}$ region between 2 and $4 \mathrm{~dB}$.

\section{Acknowledgments}

The financial support of the EPSRC, UK under the auspices of the UK-India Centre of Excellence in Wireless Communications as well as that of the EPSRC under the China-UK Science Bridge in 4G Wireless Communications is gratefully acknowledged.

\section{References}

1 Wozencraft, J.M., Horstein, M.: 'Coding for two-way channels'. Technical Report, Research Laboratory of Electronics, M.I.T, 1961

2 Wozencraft, J.M., Horstein, M.: 'Digitalised communication over twoway channels'. The Fourth London Symp. of Information Theory, London, England, 29 August-3 September 1960

3 Lin, S., Yu, P.: 'A hybrid ARQ scheme with parity retransmission for error control of satellite channels', IEEE Trans. Commun., 1982, 30, pp. 1701-1719

4 Hanzo, L., Blogh, J.S., Ni, S.: '3G, HSPA and FDD versus TDD networking: smart antennas and adaptive modulation' (Wiley, February 2008, 2nd edn.)

5 IEEE: IEEE standard for local and metropolitan area networks. Part 16: air interface for fixed and mobile broadband wireless access systems, IEEE Std 802.16e ed., 2005, www.ieee.org 
6 Nguyen, T.D., Yang, L.L., Hanzo, L.: 'Systematic Luby transform codes and their soft decoding'. IEEE Workshop on Signal Processing Systems, 17-19 October 2007, pp. 67-72

7 Hanzo, L., Alamri, O., El-Hajjar, M., Wu, N.: 'Near-capacity multifunctional MIMO systems: sphere-packing, iterative detection and cooperation' (John Wiley - IEEE Press, May 2009)

8 Sendonaris, A., Erkip, E., Aazhang, B.: 'User cooperation diversity. Part I. System description', IEEE Trans. Commun., 2003, 51, pp. 1927-1938

9 Sendonaris, A., Erkip, E., Aazhang, B.: 'User cooperation diversity. Part II. Implementation aspects and performance analysis', IEEE Trans. Commun., 2003, 51, pp. 1939-1948

10 Kramer, G., Gastpar, M., Gupta, P.: 'Cooperative strategies and capacity theorems for relay networks', IEEE Trans. Inf. Theory, 2005, 51, pp. 3037-3063

11 Lai, L., Liu, K., El-Gamal, H.: 'The three-node wireless network: achievable rates and cooperation strategies', IEEE Trans. Inf. Theory, 2006, 52, pp. $805-828$

12 Dohler, M., Lefranc, E., Aghvami, H.: 'Virtual antenna arrays for future wireless mobile communication systems'. IEEE ICT 2002, Beijing, China, June 2002, [CD Rom]

13 Janani, M., Hedayat, A., Hunter, T.E., Nosratinia, A.: 'Coded cooperation in wireless communications: space-time transmission and iterative decoding', IEEE Trans. Signal Process., 2004, 52, pp. 362-371

14 Bao, X., Yu, M., Li, J.: 'A new user cooperation scheme for uplink wireless networks'. Proc. Allerton Conf. on Communications, Control and Computing, Urbana Champaign, IL, September 2004

15 Zhang, Z., Bahceci, I., Duman, T.: 'Capacity approaching codes for relay channels'. Proc. Int. Symp. on Information Theory, 2004, May ISIT 2004, 27 June-2 July 2004, p. 2

16 Ng, S.X., Li, Y., Hanzo, L.: 'Distributed turbo trellis coded modulation for cooperative communications'. Proc. IEEE Int. Conf. on Communications, ICC'09, 14-18 June 2009, pp. 1-5

17 Soijanin, E., Varnica, N., Whiting, P.: 'Punctured vs rateless codes for Hybrid ARQ'. IEEE Information Theory Workshop, 2006, ITW'06, Punta del Este., 13-17 March 2006, pp. 155-159

18 Castura, J., Mao, Y.: 'Rateless coding and relay networks', IEEE Signal Process. Mag., 2007, 24, p. 5

19 Caire, G., Tuninetti, D.: 'The throughput of hybrid-ARQ protocols for the Gaussian collision channel', IEEE Trans. Inf. Theory, 2001, 47, pp. $1871-1988$

20 Zhao, B., Valenti, M.: 'Practical relay networks: a generalization of hybrid-ARQ', IEEE J. Sel. Areas Commun., 2005, 23, pp. 7-18

21 Bonello, N., Zhang, R., Chen, S., Hanzo, L.: 'Reconfigurable rateless codes', IEEE Trans. Wirel. Commun., 2009, 8, pp. 5592-5600

22 Luby, M.: 'LT codes'. Proc. 43rd Annual IEEE Symp. on Foundations of Computer Science, 2002, 16-19 November 2002, pp. 271-280
23 Tee, R., Nguyen, T., Ng, S., Yang, L.-L., Hanzo, L.: 'Luby transform coding aided bit-interleaved coded modulation for the wireless Internet'. Proc. IEE 66th Vehicular Technology Conf., 2007, VTC-2007, Fall, 2007, 30 September 2007-3 October 2007, pp. 2025-2029

24 Tee, R.Y.S., Nguyen, T.D., Yang, L.L., Hanzo, L.: 'Serially concatenated Luby transform coding and bit-interleaved coded modulation using iteratlive decoding for the wireless Internet'. Proc. IEEE 63rd Vehicular Technology Conf. on VTC 2006, Spring, 7-10 May 2006, vol. 1, pp. 22-26

25 Palanki, R., Yedidia, J.: 'Rateless codes on noisy channels'. Proc. Int. Symp. on Information Theory, 2004, ISIT 2004, 2004, p. 38

26 Nguyen, T., Yang, L., Ng, S., Hanzo, L.: 'An optimal degree distribution design and a conditional random integer generator for the systematic Luby transform coded wireless Internet'. Wireless Communications and Networking Conf., 2008, WCNC 2008, 31 March-3 April 2008, pp. 243-248

27 ten Brink, S.: 'Convergence of iterative decoding', Electron. Lett., 1999, 35, pp. 806-808

28 Sharon, E., Ashikhmin, A., Litsyn, S.: 'Analysis of low-density paritycheck codes based on exit functions', IEEE Trans. Commun., 2006, 54, pp. 1407-1414

29 Ochiai, H., Mitran, P., Tarokh, V.: 'Design and analysis of collaborative diversity protocols for wireless sensor networks'. Proc. IEEE 60th Vehicular Technology Conf., 2004, VTC2004-Fall, 26-29 September 2004, vol. 7, pp. 4645-4649

30 Steele, R., Hanzo, L.: 'Mobile radio communications: second and thirdgeneration cellular and WATM systems' (John Wiley - IEEE Press, May 1999, 2nd edn.)

31 Lin, S., Costello, D.J.: 'Error control coding' (Prentice Hall, 2004, 2nd edn.)

32 Valenti, M.C., Zhao, B.: 'Hybrid-arq based intra-cluster geographic relaying'. Proc. IEEE Military Communications Conf. MILCOM 2004, 31 October-3 November 2004, vol. 2, pp. 805-811

33 Host-Madsen, A., Zhang, J.: 'Capacity bounds and power allocation for wireless relay channels', IEEE Trans. Inf. Theory, 2005, 51, pp. 2020-2040

34 Lee, W.C.Y.: 'Estimate of channel capacity in rayleigh fading environment', IEEE Trans. Veh. Technol., 1990, 39, pp. 187-189

35 Fan, Y., Wang, C., Thompson, J., Poor, H.V.: 'Recovering multiplexing loss through successive relaying using repetition coding', IEEE Trans. Wirel. Commun., 2007, 6, pp. 4484-4493

36 Kong, L., Ng, S.X., Maunder, R., Hanzo, L.: 'Maximum-throughput irregular distributed space-time code for near-capacity cooperative communications', IEEE Trans. Veh. Technol., 2010, 59, pp. $1511-1517$ 